\title{
Imatinib independent aberrant methylation of NOV/CCN3 in chronic myelogenous leukemia patients: a mechanism upstream of BCR-ABL1 function?
}

\author{
Mousa Vatanmakanian, Mahmood Tavallaie and Shirin Ghadami*
}

\begin{abstract}
Background: The NOV gene product, $C \mathrm{CN} 3$, has been reported in a diverse range of tumors to serve as a negative growth regulator, while acting as a tumor suppressor in Chronic Myelogenous Leukemia (CML). However, the precise mechanism of its silencing in CML is poorly understood. In the current study, we aimed to query if the gene regulation of CCN3 is mediated by the promoter methylation in the patients with CML. In addition, to clarify whether the epigenetic silencing is affected by BCR-ABL1 inhibition, we assessed the methylation status in the patients at different time intervals following the tyrosine kinase inhibition using imatinib therapy, as the first-line treatment for this type of leukemia.

Methods: To address this issue, we applied bisulfite-sequencing technique as a high-resolution method to study the regulatory segment of the CCN3 gene. The results were analyzed in newly diagnosed CML patients as well as following imatinib therapy. We also evaluated the correlation of CCN3 promoter methylation with BCR-ABL1 levels.

Results: Our findings revealed that the methylation occurs frequently in the promoter region of CML patients showing a significant increase of the methylated percentage at the $\mathrm{CpG}$ sites compared to normal individuals. Interestingly, this hypermethylation was indicated to be independent of BCR-ABL1 titers in both groups, which might suggest a mechanism beyond the BCR-ABL1 function.

Conclusion: Despite suggesting that the CCN3 hypermethylation acts as a molecular mechanism independent of BCR-ABL1 function in CML patients, this scenario requires further validation by complementary experiments. In the case of acting upstream of BCR-ABL1 signaling, the methylation marker can provide early detection and a novel platform for targeted epigenetic modifiers for efficient treatment in imatinib resistant patients.
\end{abstract}

Keywords: NOV/CCN3, DNA methylation, Chronic myelogenous leukemia (CML), BCR-ABL1

\section{Background}

Chronic Myelogenous Leukemia (CML) is categorized as a myeloproliferative disorder which constitutes approximately $25 \%$ of leukemia cases. The disease was originally discovered to be resulted from a clonal expansion of an affected hematopoietic stem cell [1-3]. The well-known triggering molecular event initiating the CML is a chromosomal translocation between chromosomes 9 and $22, \mathrm{t}(9 ; 22)$, leading to the formation of a fusion

\footnotetext{
* Correspondence: Shiringhadami@gmail.com

Human Genetics Research Center, Baqiyatallah University of Medical Sciences, Mollasadra Ave., Vanak Square, Tehran, Iran
}

onco-protein (BCR-ABL1) which is characterized by an overactive tyrosine kinase activity [4]. Much like other enzymes in this class, the ABL1 gene product is assumed to play roles in controlling cellular growth and response to DNA damage $[5,6]$. Although the precise underlying mechanism through which this alteration results in leukemogenesis is not well clarified, the ultimate event is the proliferation of the stem cell pool with uncontrolled expansion of mature cells from myeloid lineage. The association of BCR-ABL1 expression has been established with a wide range of pathways involved in cell proliferation, survival, and apoptosis resistance [7]. The upstream

(c) The Author(s). 2019 Open Access This article is distributed under the terms of the Creative Commons Attribution 4.0 International License (http://creativecommons.org/licenses/by/4.0/), which permits unrestricted use, distribution, and 
key events, however, by which the BCR-ABL1 positive stem cells dominate the bone marrow leading to CML formation is still under query. Currently, the CML cases are clinically diagnosed in chronic phase, and the onset of disease remains elusive days or years prior to detection.

Over the last two decades, much progress has been made to reveal phenotypic outcomes associated with post-translational modifications in histone, DNA modification, and non-coding RNAs as integral parts of epigenetic machinery. Notably, large cohort of studies has reported the deregulated DNA methylation in multiple genes which associates with disease progression toward malignant behaviors and more advanced clinical staging $[8,9]$. It is hypothesized that if detectable epigenetic signatures in the human genome functioning beyond the traditional culprits of cancer initiators can be revealed, they might serve as the candidate markers for disease prediction or even the prophylactic intervention using targeted epigenetic modifiers.

With respect to newer technologies, we have used bisulfite sequencing method to elucidate the DNA methylation profile of the NOV/CCN3 gene which is implicated in the clonal evolution of CML cancer stem cells. This study was aimed to evaluate whether the methylation alteration of the stem cell-associated gene, Nephroblastoma Overexpressed (NOV), occurs beyond the function of BCR-ABL fusion gene.

It has been established that Cellular Communication Network Factor 3 (CCN3) protein plays an immediate-early roles in key cellular events such as proliferation, cellular adhesion, migration, cell fate determination, and survival [10-13]. Its crucial role in modulating the functional integrity of hematopoietic stem and progenitor cells has been also well-evidenced [14]. Although acting as metastatic accelerator in many solid tumors including Ewings Sarcoma [15], osteosarcoma [16], prostate neoplasia [17], breast [18], and bladder cancer [19]; the NOV gene was shown to be silenced in CML serving as a tumor suppressor and growth regulator [20-22]. Nevertheless, the underlying mechanism by which the gene is down-regulated is partially elucidated [20, 22].

Enrichment of DNA methylation in the CpG segments is a well-known mechanism influencing gene promoter and regulatory regions. This epigenetic-based gene silencing mostly contributes to inactivate the tumor suppressor genes in malignant cells [23, 24]. Owing to the previous data on the contribution of CCN3 during the HSC maintenance as well as the lower expression of the gene in CML, we were wondering whether the NOV gene is controlled via promoter methylation. Our study was designed also to address if the possible changes in DNA methylation of NOV gene remain intact following the imatinib treatment as a BCR-ABL1 suppressor. Although the evidence provided by our study might not be adequate to clearly estimate the hierarchical events during BCR-ABL1-positive HSCs domination, we revealed that hypermethylation that occurs in $\mathrm{CML}$ patients is not influenced by targeting the BCR-ABL1 tyrosine kinase.

\section{Methods}

\section{Patients and sample collection}

The peripheral blood samples were collected in tubes containing K2-EDTA as anti-coagulant from 20 newly diagnosed CML patients between October 2017 and July 2018 (median age of 49 years, ranging from 21 to 75 years; male: 9; female: 11) in the Gholhak Laboratory, Tehran, Iran approved by the institutional standard protocols. The diagnosis was performed based on morphology, chromosome analysis using karyotyping which was definitely confirmed by the measurement of BCR-ABL1 fusion gene titers using absolute quantification using real-time PCR. Nine patients were classified in chronic phase, 5 in accelerated phase and 6 in blastic phase. A written consent was provided for each individual participant and the study was approved by the Medical Ethics Committee of Baqiyatallah University of Medical Sciences.

\section{Molecular and hematologic response evaluation}

The subjects were then followed up for 11 months following treatment and the second sample was collected from the participants at various time intervals of imatinib therapy (6-11 months). Twenty five samples were also taken from healthy individuals as a control group and confirmed to have normal $\mathrm{CBC}$ and negative for BCR-ABL1 fusion gene transcripts. The imatinib mesylate dosages were administered for the patients based on hematologic and non-hematologic toxicities, ranging from 200 to $400 \mathrm{mg}$ P.O. daily for chronic phase, 300800 for accelerated phase, and $600-800$ for blastic phase. The Complete Hematologic Responses (CHR) were evaluated as defined by the WBC count bellow $10 \times 10^{9} / \mathrm{L}$, the absence of immature cells (including blast cells, myelocytes, and promyelocytes) using microscopic assessment of peripheral blood, and undetectible signs of leukemia such as palpable splenomegaly lasting for at least 4 weeks. In addition, an undetectable BCR-ABL/ ABL transcripts was defined as a Complete Molecular Response (CMR), while 3-log or more decline in titers compared to the baseline levels defines a Major Molecular Response (MMR). No Molecular Response (NMR) was also defined as detecting increasing changes in BCR-ABL/ABL compared with baseline; and a Partial Molecular Response (PMR) was considered as a reduction in BCR-ABL/ABL transcript less than 3-log. 


\section{DNA extraction and bisulfite conversion}

The genomic DNA samples were isolated from the peripheral blood using the KBC Blood DNA Extraction Kit with the standard salting out/proteinase $\mathrm{K}$ method (Kosar Biotechnology Co. Tehran, Iran). The samples were then quantified using a Nano-drop spectrophotometer (Thermofisher Scientific) and ensured to have a high concentration and purity, which is optimal for efficient bisulfite conversion (the concentration of more than $200 \mathrm{ng} / \mu \mathrm{l}$, OD260/230 of $2.0-2.2$; and 260/280 of 1.8-2.0). The DNA samples were then treated with sodium bisulfite according to the manufacturer's instructions (EpiTect Bisulfite Kit, QIAGEN, Hilden, Germany). The bisulfite treatment catalyzes the deamination of all the unmethylated cytosine $(\mathrm{uC})$ nucleotides to uracil (U) or thymidine $(\mathrm{T})$ nucleotides and leaves the methylated cytosine $(\mathrm{mC})$ unchanged. For optimal results, we input the amount of starting DNA from 200 to $500 \mathrm{ng}$ in the bisulfite modification process.

\section{Primer design and bisulfite sequencing PCR (BSP)}

The MethPrimer tool (http://www.urogene.org/cgi-bin/ methprimer/methprimer.cgi) was used to predict the CpG Island based on the promoter sequence provided from UCSC database, and BSP primers were designed according to the standard parameters and synthesized by GenFanAvaran Co. (Tehran, Iran). The PCR reactions were performed using $12 \mu \mathrm{l}$ of Taq DNA Polymerase 2x Master Mix RED with $2 \mathrm{mM} \mathrm{MgCl} 2$ final concentration (Ampliqon, Odense M, Denmark); $2 \mu$ l bisulfite treated DNA (approximately $50 \mathrm{ng}$ ), $0.5 \mu \mathrm{l}$ of each forward and reverse primers $(10 \mathrm{pM})$, and $\mathrm{ddH} 2 \mathrm{O}$ to the final volume of $25 \mu$ l. The reactions were conducted in a SimpliAmp thermal cycler device (Applied Biosystems) with a primary denaturation for $5 \mathrm{~min}$ at $95^{\circ} \mathrm{C}$ followed by 40 cycles of denaturation at $95^{\circ} \mathrm{C}$ for $30 \mathrm{Sec}$, annealing temperature with descending touchdown system $\left(-0.2^{\circ} \mathrm{C}\right)$ starting at $59^{\circ} \mathrm{C}$ for $45 \mathrm{Sec}$, an extension at $72^{\circ} \mathrm{C}$ for 45 $\mathrm{Sec}$; and a final extension at $72{ }^{\circ} \mathrm{C}$ for $10 \mathrm{~min}$. Following DNA cleanup procedure using the shrimp alkaline phosphatase enzyme for PCR product purification (Thermofisher Scientific). The products were then sequenced with an ABI PRISM BigDye terminator sequencing kit v1.1 (Life Technologies), and directly analyzed by an automated ABI 3130 Genetic Analyzer (Life Technologies).

\section{Data interpretation and statistical analysis}

Sequence alignment, methylation analysis, and interpretation were performed using the BiQ Analyzer software v2.02 [25]. The statistical analysis and lollipop-style representation of methylation data were provided using the QUMA online tool ((http://quma.cdb.riken.jp/). Accordingly, as the methylation status does not distribute normally, particularly in case of hypo- and hyper- methylation, the significance of difference between two sets of entire CpG sites in BSP groups is determined by the Mann-Whitney U-test which is a non-parametric test for evaluation of samples with such distributions. Also, the difference between two BSP groups at each CpG site is measured by the Fisher's exact test as non-parametric statistical significance test to assess whether nonrandom correlation exists between two categorical data. The $p$-value showing the independence of CpG methylation between two CpG sites was calculated from Fisher's exact test and the results of less than 0.05 were considered statistically significant. The statistical analysis of numeric data to determine other correlations were implemented using the SPSS 20.0 software package (SPSS, Chicago, IL, USA).

\section{Results}

Two CpG islands were predicted using the MethPrimer tool in proximity of the transcriptional start site of the NOV gene. A schematic representation of the predicted islands and the position of the primers are depicted in Fig. 1a. The island 1 was positioned at nt -200 to +20 and Island 2 at nt +41 to +131 related to the first exon. The primer was designed to create an amplicon spanning all the exon 1 and a part of promoter (both prone to be regulated by DNA methylation). CpG rich region with $16 \mathrm{CpG}$ sites from $\mathrm{nt}-16 \mathrm{bp}$ to $+255 \mathrm{bp}$ resulting in a product with $271 \mathrm{bp}$ was considered as the region for designing BSP specific primers, NOV-F1: 5' AAGG AGAGTAGTATTTATAGTTAATTGTTA-3', NOV-R1: 5' - ATACACCAAAATAATACAAAATCAC-3'. The target $\mathrm{PCR}$ sequenced region and the $\mathrm{CPG}$ positions are shown below:

\section{aaggagagcagcaccacagccaattgccatggcaaccc CGg gttCGttccacttcccacccagcCGatctccccctcctcctgcactgca gccaacCGgcttgtgCGCGtccaggagCGCGctataaaacc tgtgctgggCGtgatCGgcaagcacCGgaccagggggaaggCGag cagtgccaatctacagCGaagaaagtctCGtttggtaaaagCGagaggg gaaagctgagctgCGtgactctgtattactttggtgcat.}

\section{The CML patinets show a hypermethylated NOV gene compared to normal individuals}

According to analysis by BSP and sequencing interpretation, the NOV gene was shown to be significantly hypermethylated in CML patinets compared to healthy individuals. The $\mathrm{CpG}$ methylation percentage in the CML patinets in chronic (60.8\%), accelerated (75.9\%), and blastic (66.7\%) phases were significantly greater than those observed in normal subjects (34.8\%) (Mann Wittney U-test: $P=0.0001)$. The lollipops plot of methylated and unmethylated $\mathrm{CpG}$ sites as well as the aggregated representation of the methylation data are illustrated in Fig. 2 and Fig. 3, respectively. 
A

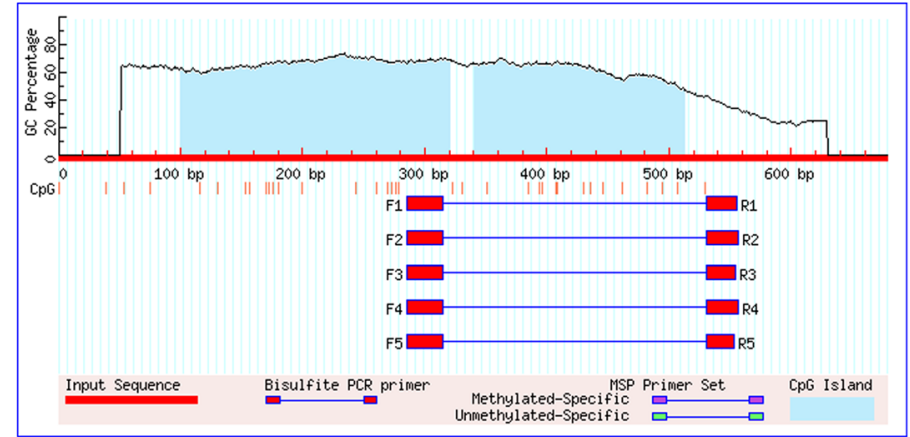

B

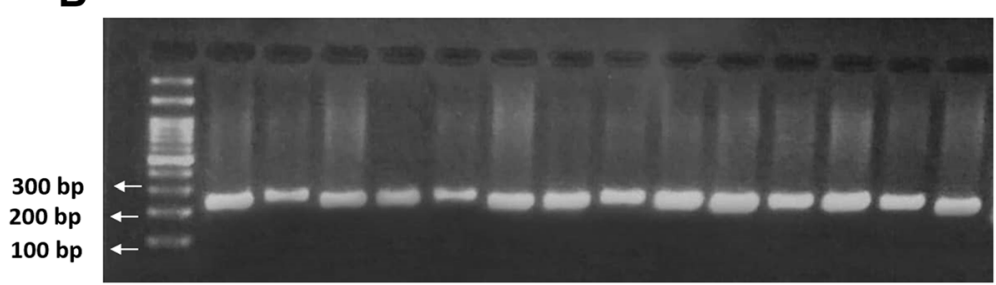

Fig. 1 Schematic diagram of the analyzed BSP primer. a: The schematic representation of the predicted CpG islands (blue region), CpG sites (small red bars), and Bisulfite PCR primer positions (F1: Forward primer; R1: Reverse primer). b: The PCR bands of the samples following the NOV amplification showing the product size of $271 \mathrm{bp}$

The methylation of CpG island was observed in $66.4 \%$ of CpG sites (ranging from 12.5 to $87.5 \%$; median: $75 \%$ ) of samples obtained from CML patients and 34.8\% (ranging from 12.5 to $75 \%$; median: $25 \%$ ) of control samples. Collectively, $6 \mathrm{CpG}$ positions showed a significant hypermethylation in CML group ( $P$-value of Fisher's exact test less than 0.05 ) including the $\mathrm{CpG}$ number $1(P=0.008), 8(P=0.0003), 9$ $(P=0.0008), 10(\mathrm{P}=0.0003), 11(\mathrm{P}=0.000), 14(P=0.001)$.

\section{The NOV methylation pattern remains intact following imatinib therapy in CML patients}

To determine whether the methylation pattern of NOV gene is affected by the suppression of BCR-ABL1 tyrosine kinase activity, we performed the BSP on the patients upon undergoing imatinib therapy. Our BSP analysis showed the mean methylation percentage of $68.5 \%$ for the patients undergone imatinib therapy ranging from 25 to $93.8 \%$ (mean before treatment $=66.4 \%$ ) (Fig. 4). Interestingly, our findings demonstrated that while the BCR-ABL1 titers are significantly down-regulated in the patients undergoing imatinib therapy $(P=0.011)$, the NOV methylation patterns remained intact $(P=0.978)$, suggesting that the methylation status of the NOV gene might be an independent risk factor in the CML patients which is not influenced by the BCR-ABL1 downstream events.

\section{CML patients in different clinical staging do not show variation in NOV methylation}

In order to decipher the variation in the methylation status of the NOV gene in the patients with CML in chronic, accelerated, and blastic phases, we performed the Mann-Whitney U-test between the different groups. A comprehensive statistical analysis of the methylation pattern of NOV gene is described in Table 1 for different groups of CML patients. Briefly, it was revealed that while the patients in chronic $(n=9)$, accelerated $(n=5)$, and blastic $(n=6)$ phases are significantly rich in methylated CpG sites compared to the control samples (with $P$ value $=0.029, P=$ 0.0008 , and $P=0.0032$, respectively); the statistical data indicated that regardless of the clinical staging, the patients in different CML phases with various BCR-ABL1 titers are not significantly different in terms of NOV methylation levels ( $\mathrm{Pv}$ of chronic-accelerated analysis $=0.72$; chronic-blastic $=$ 0.93 ; and accelerated-blastic $=0.61$ ), which further validates the assumption that NOV methylation might act as an independent factor during CML pathogenesis.

\section{Clinical and laboratory characteristics of CML patients}

Of the Nine patients in chronic phase, 8 (88.8\%) achieved a Complete Hematologic Response (CHR); while 5 (55.5\%) showed a Complete Molecular Response (CMR), 3 (33.3\%) achieved Partial Molecular Response (PMR), and 1 (11.1\%) with increased BCR-ABL/ABL ratio (NMR) within 6 months of follow up. In the patients with accelerated phase, 3 cases (60\%) achieved CHR, and $2(40 \%)$ gained MMR within 11 months of follow up. The patients in blastic phase showed a poorer response, 3 showed the CHR (50\%), whereas 2 (33.3\%) achieved the MMR, 3 (50\%) with PMR, and 1 (16.6\%) with NMR. In general, the hematologic and molecular 


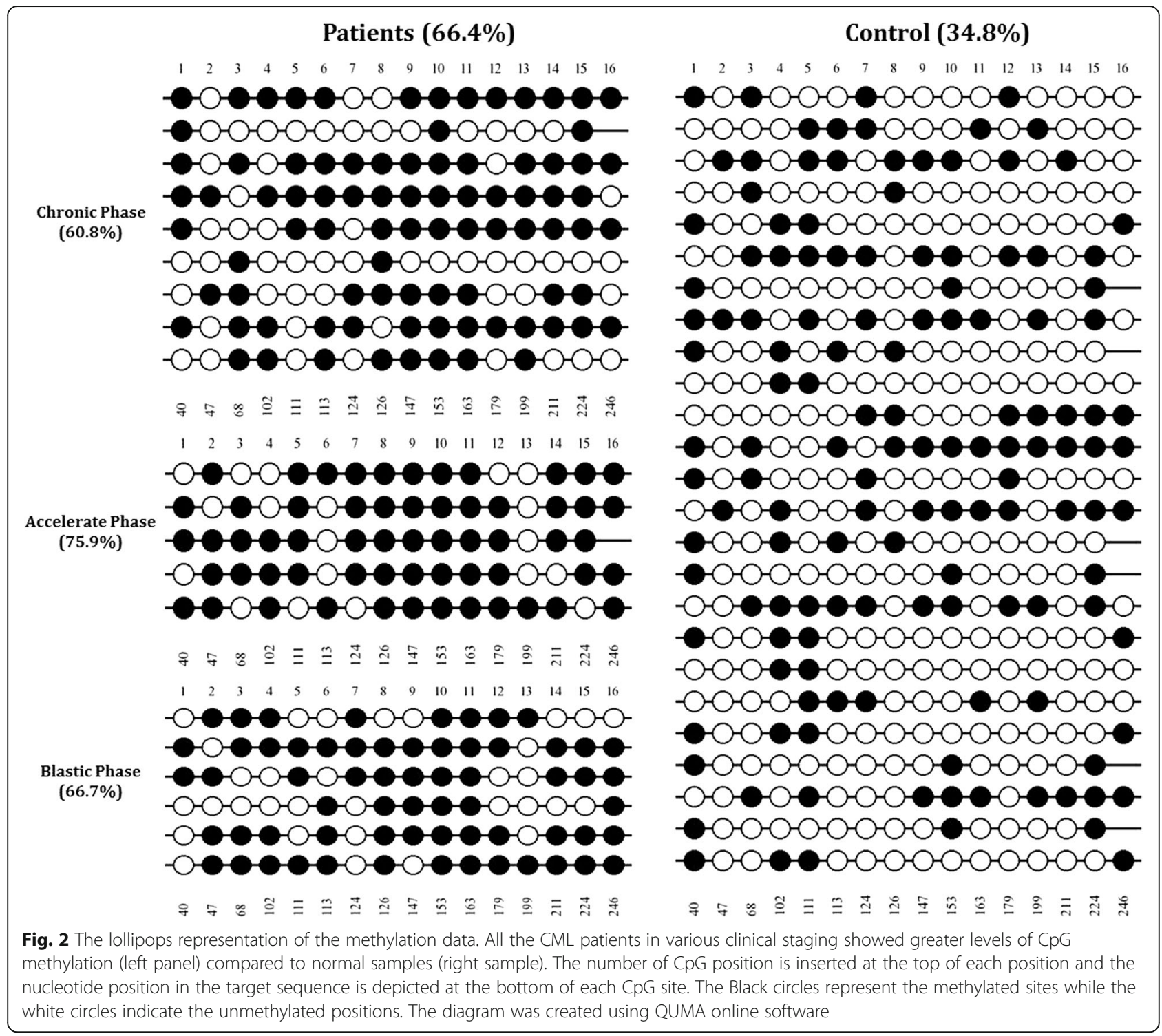

responses showed no significant correlation $(P=$ 0.085). Also, no meaningful relationship was found between the patient's sex, dosage, or duration of imainib consumption $(p=1.10, p=0.65, p>0.05)$. Interestingly, regardless of clinical staging and patient's response to imatinib treatment, the analysis of NOV promoter showed no significant changes in methylation level among the treated patients.

\section{Discussion}

The presence of the BCR-ABL1 fusion gene as a pathognomonic molecular event in almost all the CML cases has distinguished this malignancy from other myeloproliferative disorders. In spite of significant efforts made to deciphering mechanisms of the disease pathogenesis and elucidation of many BCR-ABL molecular targets, the underlying mechanisms triggering the disease and those responsible for clonal evolution of BCR-ABL-positive clones have not been comprehensively clarified.

Having the integral roles during clonal evolution in leukemia [26-28], the epigenetic mechanisms are considered as ideal candidates to be analyzed for scrutinizing the process of the leukemia initiation and progression. Therefore, we sought to investigate the possible involvement of DNA methylation as the well-known component of the epigenetic machine in the pathogenesis of CML patients in a group of clinical samples confirmed to have CML. Our study revealed that $\mathrm{CCN} 3 / \mathrm{NOV}$, as a key regulator in CML, significantly hypermethylated in the patients compared to normal individuals. By analyzing the clinical samples collected from the different clinical staging of CML patients and also measuring the methylation levels following imatinib therapy, we further validated that the $\mathrm{CCN} 3$ remains 


\section{CML patients}

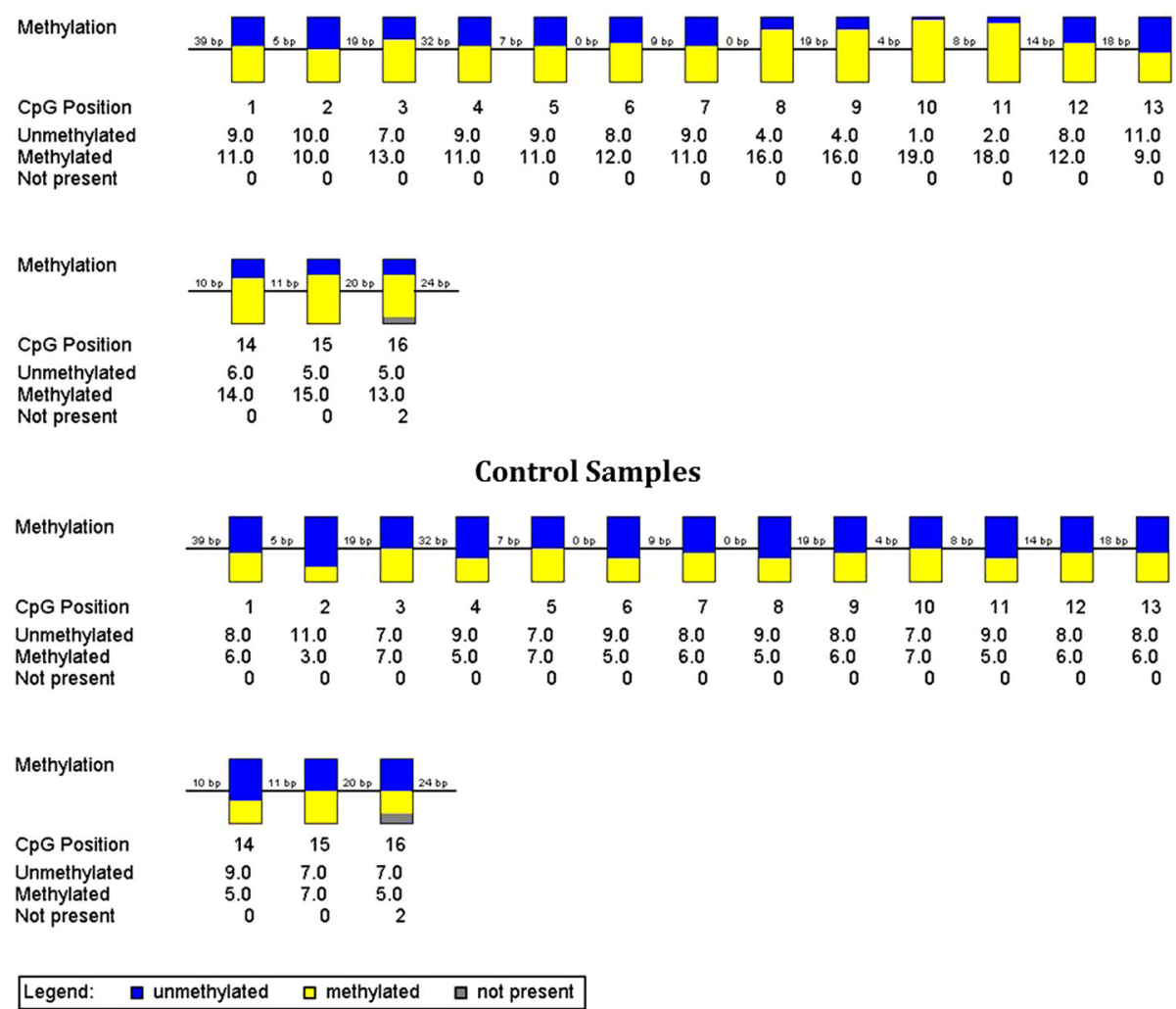

Fig. 3 The aggregated representation of methylation data. The Methylation analysis was performed on BSP results for 20 CML patients in different clinical staging and 25 healthy individuals. The relative position of CpG sites is depicted by the gaps between the boxes. The graph was drawn using BiQ Analyzer software v 2.02

hypermethylated in the patients following treatment with BCR-ABL1 tyrosine kinase inhibitor, imatinib; as well as in different clinical stages. These findings raise the question that DNA methylation of $\mathrm{CCN} 3$ might serve as the molecular event acting upstream of BCR-ABL1 functions. However, supporting this idea requires further efforts analyzing functional consequences using standard experimental platforms.

BCR-ABL1 formation seems to be an early event during CML pathogenesis which also governs numbers of downstream molecular deregulations responsible for cell proliferation, apoptosis resistance, survival, and cellcycle progression, thus affecting the overall disease progression [29]. Despite this fact, it has been proposed that there might be the epigenetic changes which decide which clones will dominate the stem cell niche and evolve to differentiated functioning cells [30].

Nephroblastoma Overexpressed (NOV) encodes a small secreted protein, which is a member of the CCN family of regulator proteins (CCN3). This family has been reported to regulate the skeletal and cardiovascular development, fibrosis, as well as cancer formation. Based on Gene Ontology (GO) annotations, the association of
CCN3 in Notch signaling pathway (KEGG) and cell adhesion has been also described in which it serves a growth factor activity and integrin binding [31].

Gupta et al. reported that the functional integrity of hematopoietic stem and progenitor cells is regulated in large extent by the matricellular protein CCN3 [14]. They showed that the forced expression of recombinant Nov accelerates either the primitive stem or progenitor activity. Their findings supported the idea that CCN3 is an essential regulator of human HSC or progenitor cells.

A previous study introduced the $\mathrm{CCN} 3$ as a novel gene to be downregulated in CML cells. It was also supported that this suppression is a direct consequence of BCR-ABL1 kinase activity. This was shown to be a novel downstream event for BCR-ABL activity. Briefly, Lynn McCallum et al. discuss that cellular CCN3 is decreased in the BCR-ABL active FDCB-Mix cells. They also demonstrated that targeting $\mathrm{BCR}-\mathrm{ABL}$ kinase activity by imatinib or siRNA significantly inhibits the BCR-ABL while increasing the CCN3 levels. Hence, they came to the conclusion that BCR-ABL directly regulates the CCN3 secretion in the CML cells where the levels of cellular CCN3 is reduced. They showed that the level of 


\section{Before Treatment (66.4\%)}

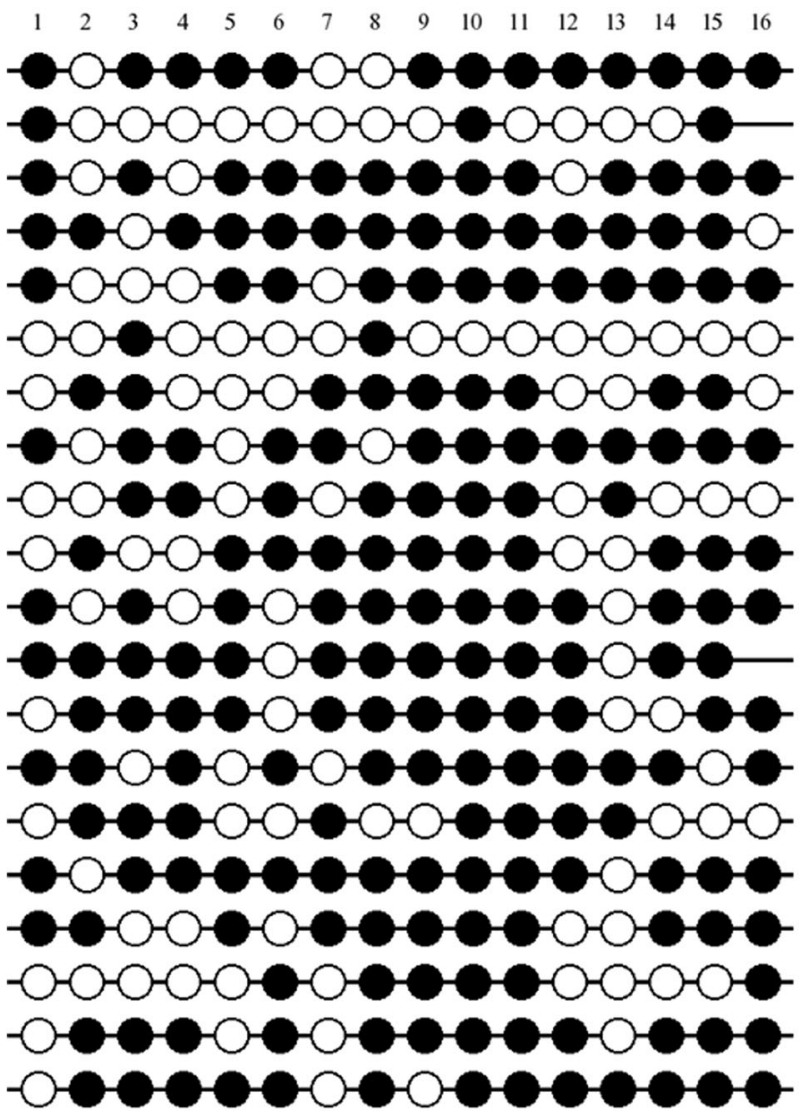

After Treatment (68.5\%)

$\begin{array}{llllllllllllllll}1 & 2 & 3 & 4 & 5 & 6 & 7 & 8 & 9 & 10 & 11 & 12 & 13 & 14 & 15 & 16\end{array}$

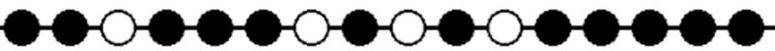

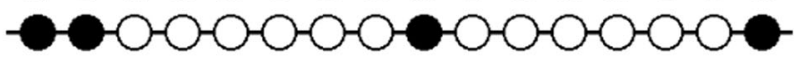

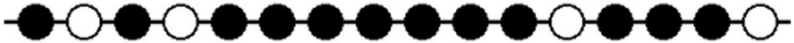

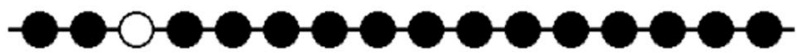

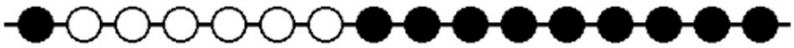

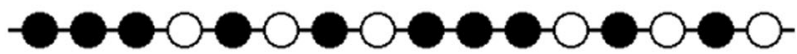

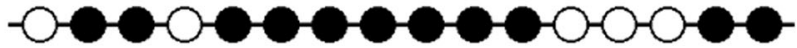

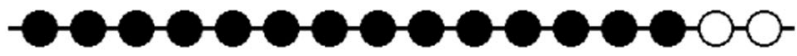

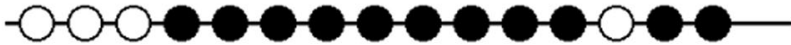
-00-00-00-00-00-0-

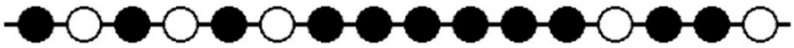

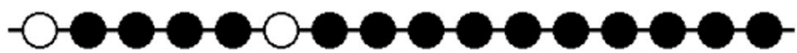

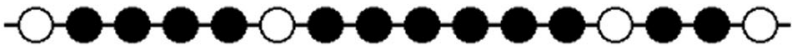

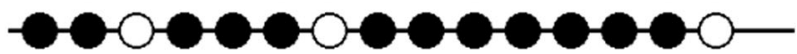

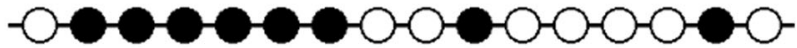

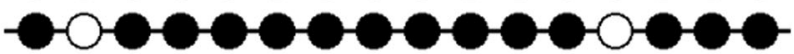

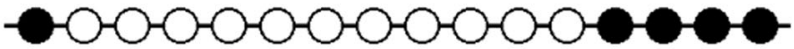

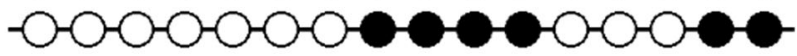

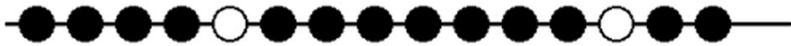
$-0-000-000-000-0$

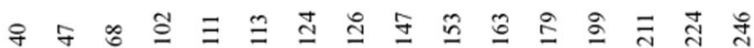

Fig. 4 The lollipops representation of the methylation pattern of patients before and after imatinib therapy. The number of CpG position is inserted at the top of each position and the nucleotide position in the target sequence is depicted at the bottom of each CpG site. The Black circles represent the methylated sites while the white circles indicate the unmethylated positions. The diagram was created using QUMA online software

secretory CCN3 following imatinib therapy not only was not reduced but also increased in extends. Our findings are consistent with this data, in which we have shown that the imatinib therapy did not affect the levels of CCN3 gene hypermethylation, a possible mechanism for the gene suppression. In case of hierarchical events, our findings might be in contrast to those obtained by the McCallum's study. However, the gene expression can be regulated at various molecular levels. Interestingly, they also transfected the CCN3 into the BCR-ABL+ cells, and observed that the stem cell proliferation and clonogenic potential were significantly suppressed. While the CCN3 regulation was considered as a downstream event of $\mathrm{BCR}-\mathrm{ABL}$ in their study, we suggest that there might be mechanisms acting beyond the $\mathrm{BCR}-\mathrm{ABL}$ function to prone the BCR-ABL+ clones dominating the stem cell niche and thereby progress to CML. We observed that even the patients with sub-optimal molecular responses (less than 3-log decrease in BCR-ABL1 transcripts compared to the baseline standard levels) or no molecular response showed the NOV hypermethylation, and no significant changes was observed in the level of NOV methylation in various clinical staging with the treatment response. However, the McCallum's results showed that the $\mathrm{CCN} 3$ expression was up-regulated following BCR-ABL inhibition. This might be interpreted as a non-methylation mechanism for $\mathrm{CCN} 3$ reactivation in this context. In addition, although the McCallum's study argues that the CCN3 secretion is controlled by direct activity of $\mathrm{BCR}-\mathrm{ABL}$, they verify the downregulated levels of cellular CCN3 in CML cells. Therefore, it can be assumed that the secretory and cellular CCN3 might be regulated through different mechanisms at distinct cellular levels [20].

A distinct study reported that despite the undetectable CCN3 levels in CML stem cells, the progenitor cells 


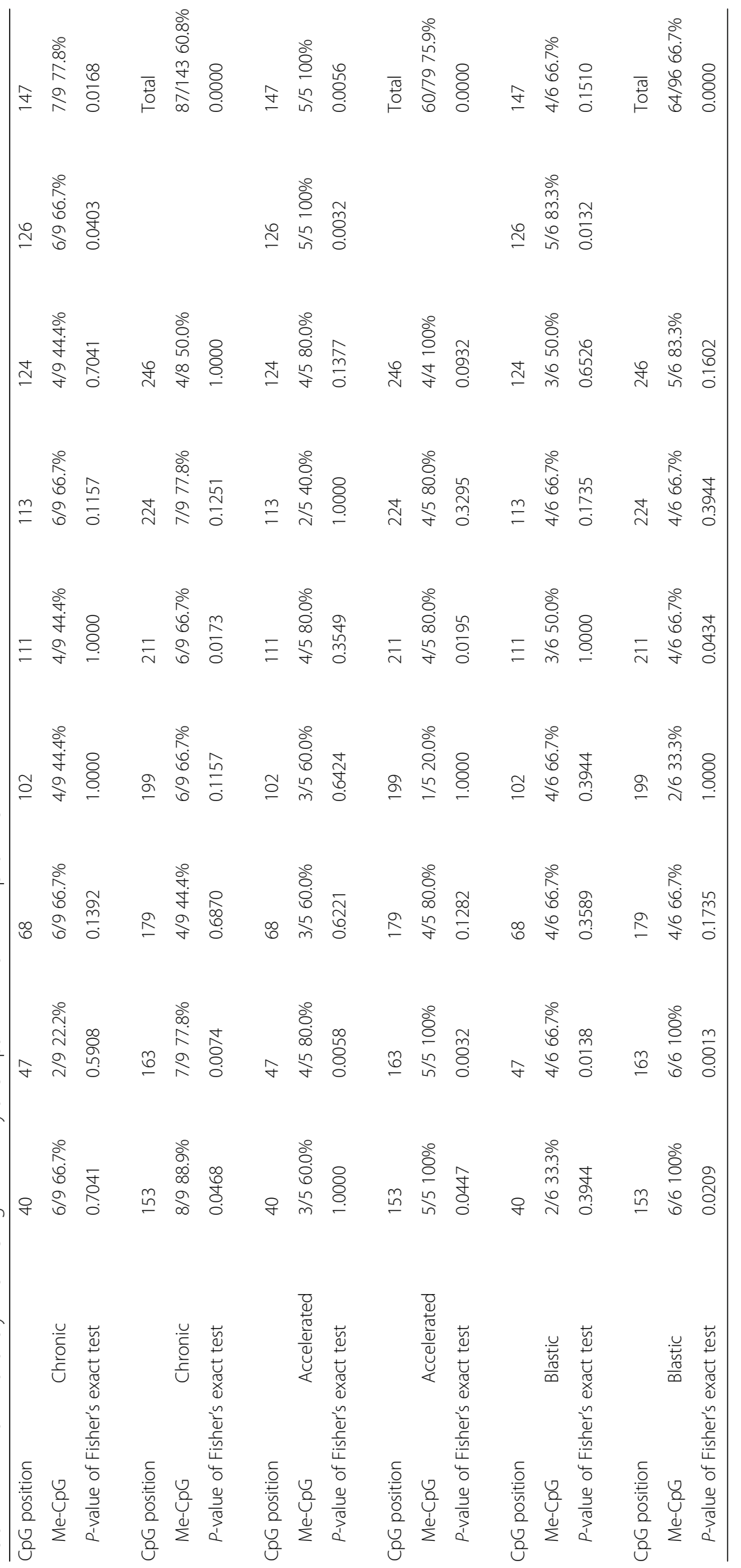


respond to recombinant $\mathrm{CCN} 3$. In this study McCallum et al. showed that abrogation of CCN3 accelerates the leukemogenesis mediated by BCR-ABL, highlighting the important growth regulatory function of $\mathrm{CCN} 3$ in hematopoiesis [21].

In another attempt to clarify the mechanism of CCN3 downregulation in CML, Suresh et al. assessed the role of MicroRNAs during BCR-ABL mediated signaling pathway. They hypothesized that BCR-ABL regulates a range of oncogenic and tumor suppressor miRNAs and thereby favors the malignant behaviors. They reported that miR-130a can significantly suppress both the levels of CCN3 mRNA and protein in HL60 cells. It was concluded that miRNA profile which is deregulated as a result of BCR-ABL activities can be one of the mechanisms for down-regulation of $\mathrm{CCN} 3$ through which the leukemic cells evade the negative growth regulation [22].

In a distinct study, McCallum et al. assessed more deeply the role of $\mathrm{CCN} 3$ during the leukemogenic process. They validated that CCN3-expressing K562 cells significantly reduce the cell growth, colony forming capacity, and mitogenic signaling while compared with the cells transfected with empty vectors. They also indicated that the CCN3 overexpressed cells accelerated the apoptosis through 3-fold increase in annexin $\mathrm{V}$ and affirmed that this apoptosis induction remains even after imatinib treatment. These findings, combined with other results showing that the transfected cells display more robust adhesive potentials highlighted the role of $\mathrm{CCN} 3$ as a growth regulator which also sensitizes the CML cells to imatinib-induced apoptosis. Their findings supported the idea that $\mathrm{CCN} 3$ can open novel doors for alternate anti-leukemia therapeutics [32].

Although recent studies have shown distinct mechanisms for $\mathrm{CCN}$ downregulation in CML and that this inhibition occurs as a direct BCR-ABL kinase activity, the epigenetic mechanisms for CCN3 suppression had been poorly understood. In the current study, we further demonstrated that deregulation of this gene might be at least in part due to hypermethylation of the promoter, which can act as an early event during the leukemogenesis. Having clonogenic activities and being involved in several properties of hematopoietic stem cells, we assume the CCN3 hypermethylation as a candidate initiator of CML, which can provide the clonal domination of BCR-ABL+ cells. However, further studies are required to clarify the exact influence of NOV DNA hypermethylation by analyzing a comprehensive set of signaling pathways involved in the disease.

\section{Conclusions}

Collectively, our findings validated a hypermethylated pattern for NOV/CCN3 gene in CML patients, which is not influenced by the imatinib therapy, suggesting this as a BCR-ABL independent molecular event, which raises the question that whether the $\mathrm{CCN} 3$ hypermethylation serves as an early trigger for CML formation. Based on our findings and previous results from other studies regarding the role of $\mathrm{CCN} 3$ in stem cell function and clonal evolution, this molecular target can provide alternative therapeutic strategies for CML management.

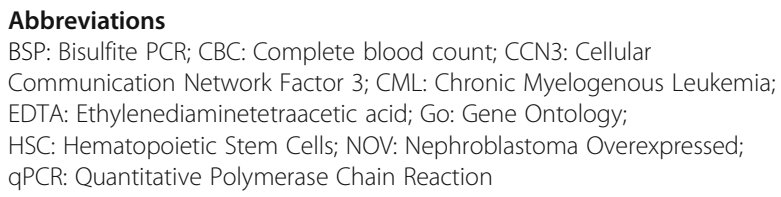

\section{Acknowledgements}

The authors have special thanks to the Baqiyatallah University of Medical Sciences Research Committee for its financial support (Grant Number: 91002804). We also grateful to Mr. Ebrahim Kiani for providing us with sequencing results, and to Director board of Gholhak Laboratory, Tehran, for their kind collaboration regarding the patients' samples, BCR-ABL1 titers and $\mathrm{CBC}$ results.

\section{Funding}

Baqiyatallah University of Medical Sciences, Deputy of Research, Tehran, Iran; Grant Number: 91002804.

\section{Availability of data and materials}

All the resulting data and the used materials are available in the main article context.

\section{Authors' contributions}

Mr. MV was responsible for performing the experiments, analyzing the data and providing the first manuscript draft. Dr. MT contributed in this research by providing the grant proposal, providing materials, and revising the final manuscript. Dr. SG designed and leaded the experiments and was responsible for designing the primers and bioinformatics studies. All authors read and approved the final manuscript.

\section{Ethics approval and consent to participate}

A written consent was provided for each individual participant and the study was approved by the Medical Ethics Committee of Baqiyatallah University of Medical Sciences.

Consent for publication

Not applicable.

\section{Competing interests}

The authors declare no conflicts of interests.

\section{Publisher's Note}

Springer Nature remains neutral with regard to jurisdictional claims in published maps and institutional affiliations.

Received: 6 February 2019 Accepted: 3 April 2019

Published online: 23 April 2019

\section{References}

1. Fialkow PJ, Martin PJ, Najfeld V, Penfold GK, Jacobson RJ, Hansen JA. Evidence for a multistep pathogenesis of chronic myelogenous leukemia. Blood. 1981;58(1):158-63.

2. Martin PJ, Najfeld V, Hansen JA, Penfold GK, Jacobson RJ, Fialkow PJ. Involvement of the B-lymphoid system in chronic myelogenous leukaemia. Nature. 1980;287(5777):49.

3. Golde DW, Burgaleta C, Sparkes R, Cline M. The Philadelphia chromosome in human macrophages. Blood. 1977;49(3):367-70. 
4. Deininger MW, Goldman JM, Melo JV. The molecular biology of chronic myeloid leukemia. Blood. 2000;96(10):3343-56.

5. Wang JY. Abl tyrosine kinase in signal transduction and cell-cycle regulation. Curr Opin Genet Dev. 1993;3(1):35-43.

6. Shafman T, Khanna KK, Kedar P, Spring K, Kozlov S, Yen T, et al. Interaction between ATM protein and c-Abl in response to DNA damage. Nature. 1997; 387(6632):520.

7. Horita M, Andreu EI, Benito A, Arbona C, Sanz C, Benet I, et al. Blockade of the Bcr-Abl kinase activity induces apoptosis of chronic myelogenous leukemia cells by suppressing signal transducer and activator of transcription 5-dependent expression of BCl-xL. J Exp Med. 2000;191(6):977-84.

8. Jelinek J, Gharibyan V, Estecio MR, Kondo K, He R, Chung W, et al. Aberrant DNA methylation is associated with disease progression, resistance to imatinib and shortened survival in chronic myelogenous leukemia. PLoS One. 2011;6(7):e22110

9. Rahmani T, Azad M, Chahardouli B, Nasiri H, Vatanmakanian M, Kaviani S. Patterns of DNMT1 promoter methylation in patients with acute lymphoblastic leukemia. International journal of hematology-oncology and stem cell research. 2017;11(3):172

10. Gellhaus A, Dong X, Propson S, Maass K, Klein-Hitpass L, Kibschull M, et al. Connexin43 interacts with NOV a possible mechanism for negative regulation of cell growth in choriocarcinoma cells. J Biol Chem. 2004; 279(35):36931-42.

11. Lin CG, Chen C-C, Leu S-J, Grzeszkiewicz TM, Lau LF. Integrin-dependent functions of the Angiogenic inducer NOV (CCN3) IMPLICATION IN WOUND HEALING. J Biol Chem. 2005;280(9):8229-37.

12. Lin CG, Leu S-J, Chen N, Tebeau CM, Lin S-X, Yeung C-Y, et al. CCN3 (NOV) is a novel angiogenic regulator of the CCN protein family. J Biol Chem. 2003;278(26):24200-8.

13. Tzeng HE, Chen JC, Tsai CH, Kuo CC, Hsu HC, Hwang WL, et al. CCN3 increases cell motility and MMP-13 expression in human chondrosarcoma through integrin-dependent pathway. J Cell Physiol. 2011;226(12):3181-9.

14. Gupta R, Hong D, Iborra F, Sarno S, Enver T. NOV (CCN3) functions as a regulator of human hematopoietic stem or progenitor cells. Science. 2007: 316(5824):590-3.

15. Fu CT, Bechberger JF, Ozog MA, Perbal B, Naus CC. CCN3 (NOV) interacts with connexin43 in $\mathrm{C} 6$ glioma cells: possible mechanism of connexinmediated growth suppression. J Biol Chem. 2004.

16. Perbal B, Zuntini M, Zambelli D, Serra M, Sciandra M, Cantiani L, et al. Prognostic value of CCN3 in osteosarcoma. Clin Cancer Res. 2008;14(3):701-9.

17. Maillard M, Cadot B, Ball R, Sethia K, Edwards D, Perbal B, et al. Differential expression of the ccn3 (nov) proto-oncogene in human prostate cell lines and tissues. Mol Pathol. 2001;54(4):275.

18. Ouellet V, Tiedemann K, Mourskaia A, Fong JE, Tran-Thanh D, Amir E, et al. CCN3 impairs osteoblast and stimulates osteoclast differentiation to favor breast cancer metastasis to bone. Am J Pathol. 2011;178(5):2377-88.

19. Chen J, Gao Y, Xu B, Cui X, Xu D. NOV is upregulated and promotes migration and invasion in bladder cancer. Tumor Biol. 2014;35(7):6749-55.

20. McCallum L, Price S, Planque N, Perbal B, Pierce A, Whetton AD, et al. A novel mechanism for BCR-ABL action: stimulated secretion of CCN3 is involved in growth and differentiation regulation. Blood. 2006;108(5):1716-23.

21. McCallum L, Lu W, Price S, Lazar N, Perbal B, Irvine AE. CCN3: a key growth regulator in chronic myeloid Leukaemia. Journal of cell communication and signaling. 2009;3(2):115-24.

22. Suresh S, McCallum L, Lu W, Lazar N, Perbal B, Irvine AE. MicroRNAs 130a/b are regulated by $B C R-A B L$ and downregulate expression of CCN3 in CML. Journal of cell communication and signaling. 2011;5(3):183-91.

23. Hamidi T, Singh AK, Chen T. Genetic alterations of DNA methylation machinery in human diseases. Epigenomics. 2015;7(2):247-65.

24. Sarkar D, Leung EY, Baguley BC, Finlay GJ, Askarian-Amiri ME. Epigenetic regulation in human melanoma: past and future. Epigenetics. 2015;10(2): 103-21.

25. Bock C, Reither S, Mikeska T, Paulsen M, Walter J, Lengauer T. BiQ analyzer: visualization and quality control for DNA methylation data from bisulfite sequencing. Bioinformatics. 2005;21(21):4067-8.

26. Puente XS, López-Otín C. The evolutionary biography of chronic lymphocytic leukemia. Nat Genet. 2013;45(3):229.

27. Landau DA, Carter SL, Getz G, Wu CJ. Clonal evolution in hematological malignancies and therapeutic implications. Leukemia. 2014;28(1):34.

28. Greaves M. Leukaemia'firsts' in cancer research and treatment. Nat Rev Cancer. 2016;16(3):163.
29. Asimakopoulos FA, Shteper PJ, Krichevsky S, Fibach E, Polliack A, Rachmilewitz $E$, et al. ABL1 methylation is a distinct molecular event associated with clonal evolution of chronic myeloid leukemia. Blood. 1999;94(7):2452-60.

30. Heller G, Topakian T, Altenberger C, Cerny-Reiterer S, Herndlhofer S, Ziegler $B$, et al. Next-generation sequencing identifies major DNA methylation changes during progression of $\mathrm{Ph}+$ chronic myeloid leukemia. Leukemia. 2016;30(9):1861.

31. O'Leary NA, Wright MW, Brister JR, Ciufo S, Haddad D, McVeigh R, et al. Reference sequence (RefSeq) database at NCBl: current status, taxonomic expansion, and functional annotation. Nucleic Acids Res. 2015;44(D1):D733-D45.

32. McCallum L, Lu W, Price S, Lazar N, Perbal B, Irvine AE. CCN3 suppresses mitogenic signalling and reinstates growth control mechanisms in chronic myeloid leukaemia. Journal of cell communication and signaling. 2012;6(1): $27-35$.
Ready to submit your research? Choose BMC and benefit from:

- fast, convenient online submission

- thorough peer review by experienced researchers in your field

- rapid publication on acceptance

- support for research data, including large and complex data types

- gold Open Access which fosters wider collaboration and increased citations

- maximum visibility for your research: over $100 \mathrm{M}$ website views per year

At $\mathrm{BMC}$, research is always in progress.

Learn more biomedcentral.com/submissions 